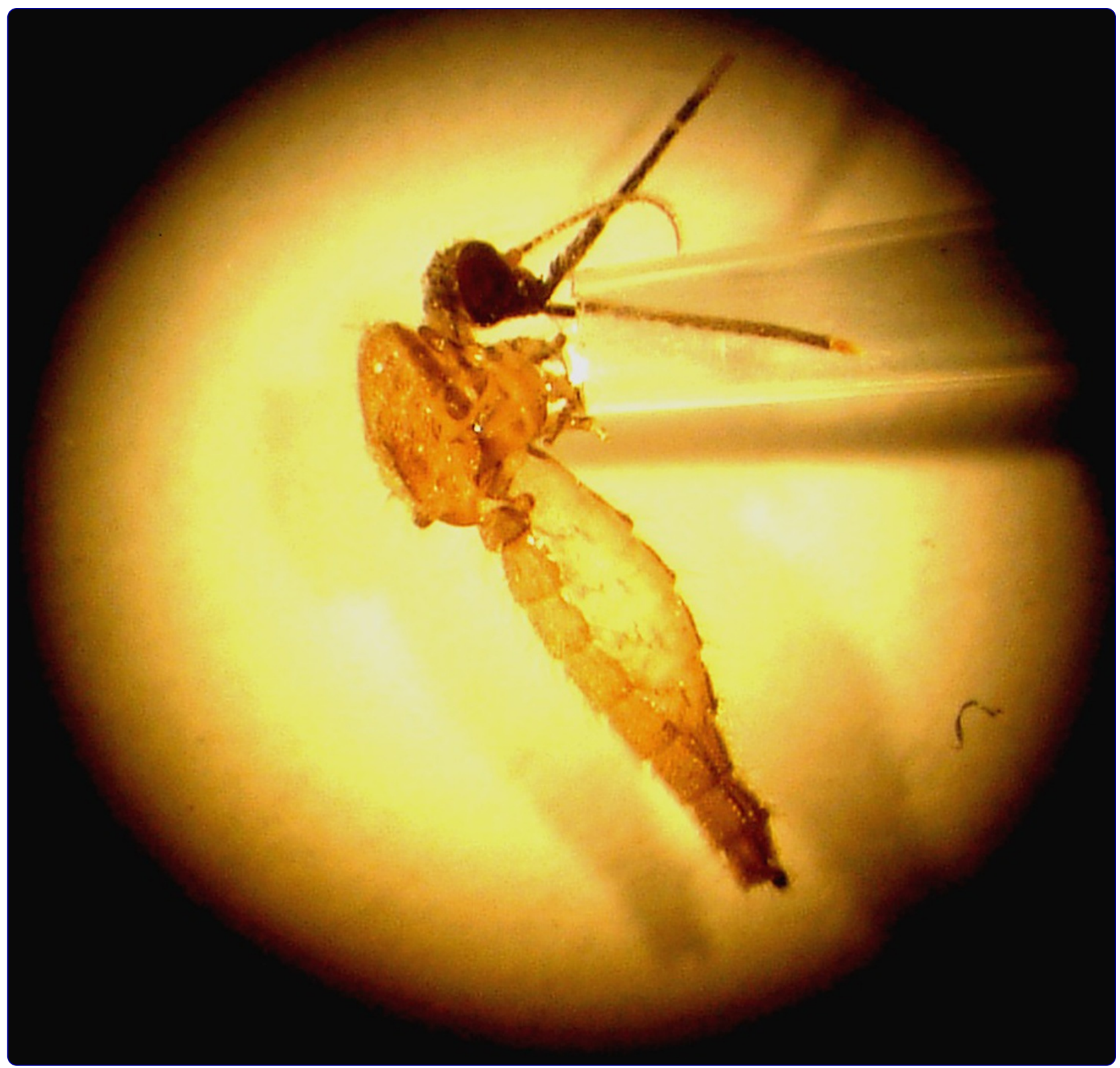

Plasmodium falciparum infection during dry season: $\lg G$ responses to Anopheles gambiae salivary gSG6-P1 peptide as sensitive biomarker for malaria risk in Northern Senegal

Sagna et al. 


\title{
Plasmodium falciparum infection during dry season: IgG responses to Anopheles gambiae salivary gSG6-P1 peptide as sensitive biomarker for malaria risk in Northern Senegal
}

André B Sagna ${ }^{1,2^{*}}$, Lobna Gaayeb ${ }^{1,3}$, Jean B Sarr ${ }^{1,4}$, Simon Senghor ${ }^{1}$, Anne Poinsignon ${ }^{4}$, Samy Boutouaba-Combe ${ }^{1}$, Anne-Marie Schacht ${ }^{1,3}$, Emmanuel Hermann $^{3}$, Ngor Faye ${ }^{2}$, Franck Remoue ${ }^{4,5}$ and Gilles Riveau ${ }^{1,3}$

\begin{abstract}
Background: The Northern part of Senegal is characterized by a low and seasonal transmission of malaria. However, some Plasmodium falciparum infections and malaria clinical cases are reported during the dry season. This study aims to assess the relationship between IgG antibody (Ab) responses to gSG6-P1 mosquito salivary peptide and the prevalence of $P$. falciparum infection in children during the dry season in the Senegal River Valley. The positive association of the Ab response to gSG6-P1, as biomarker of human exposure to Anopheles vector bite, and $P$. falciparum infectious status (uninfected, infected-asymptomatic or infected-symptomatic) will allow considering this biomarker as a potential indicator of $P$. falciparum infection risk during the dry season.

Methods: Microscopic examination of thick blood smears was performed in 371 and 310 children at the start (January) and at the end (June) of the dry season, respectively, in order to assess the prevalence of $P$. falciparum infection. Collected sera were used to evaluate IgG response to gSG6-P1 by ELISA. Association between parasitological and clinical data (infected-asymptomatic or infected-symptomatic) and the anti-gSG6-P1 IgG levels were evaluated during this period.

Results: The prevalence of $P$. falciparum infection was very low to moderate according to the studied period and was higher in January (23.5\%) compared to June (3.5\%). Specific lgG response was also different between uninfected children and asymptomatic carriers of the parasite. Children with P. falciparum infection in the dry season showed higher IgG Ab levels to gSG6-P1 than uninfected children.

Conclusions: The results strengthen the hypothesis that malaria transmission is maintained during the dry season in an area of low and seasonal transmission. The measurement of IgG responses to gSG6-P1 salivary peptide could be a pertinent indicator of human malaria reservoir or infection risk in this particular epidemiological context. This promising immunological marker could be useful for the evaluation of the risk of $P$. falciparum exposure observed during dry season and, by consequences, could be used for the survey of potential pre-elimination situation.
\end{abstract}

Keywords: Dry season, Plasmodium infection, Anti-salivary peptide, Malaria pre-elimination, Senegal River Valley, Anopheles exposure

\footnotetext{
* Correspondence: andre.sagna@espoir-sante.org

'Centre de Recherche Biomédicale Espoir Pour La Santé, 269 Route de la corniche, Sor, BP: 226, Saint-Louis, Sénégal

²Département de Biologie Animale, Laboratoire de parasitologie générale, Université Cheikh Anta Diop, Dakar, Sénégal

Full list of author information is available at the end of the article
} 


\section{Background}

Malaria transmission in the Northern part of Senegal is low and occurs mainly between August and October due to a single rainy season from July to October [1]. This situation creates breeding sites for two members of the Anopheles gambiae complex (An. gambiae s.s. and Anopheles arabiensis) and for Anopheles funestus, which breed in fresh water and in swampy habitats with much vegetation, respectively. In this area, Plasmodium falciparum is virtually the only species of Plasmodium. The incidence of clinical cases and mortality peaks between September and November, and then rapidly declines. According to statistical data of the National Malaria Control Programme (NMCP), this region of Senegal presents the lowest national malaria attack rates, with less than five malaria declared cases per 1,000 inhabitants in 2009 [2], and could, therefore, be an interesting area of study in the scope of local malaria pre-elimination.

In several epidemiologic studies, a proportion of $P$. falciparum human carriers were found during the dry season, despite a very low level of parasite transmission [3-5]. The small numbers of clinical cases of malaria observed during the middle and at the end of the dry season are considered to be mostly due to parasitological yearly recrudescence. Some infected individuals carry gametocytes throughout the dry season, potentially inducing malaria transmission if vectors are present during this period [6]. In Northern Senegal, the construction of two dams on the Senegal River has enabled the enlargement of irrigated areas and the expansion of rice growing, providing breeding sites for mosquitoes. This situation could contribute to the maintenance of malaria transmission during the dry season [7]. The identification of $P$. falciparum carriers in human populations and the evaluation of their risks of exposure to malaria vectors are needed to carry out an effective malaria control in this particular area, considered suitable for malaria pre-elimination by the NMCP.

During its blood meal, the Anopheles mosquito injects saliva into the vertebrate host. The study of salivary proteins and their effects on the immune response of vertebrate host represents an innovative research tool to characterize the mechanisms of pathogens' transmission. Therefore, the study of human-vector relationship appeared promising in the perspective of an effective control of vector-borne diseases. Numerous studies have shown that Anopheles salivary proteins are antigenic. In particular, it has been shown that children living in malaria-endemic areas developed IgG response to whole saliva of An. gambiae which was associated with the intensity of human exposure to vector bites [8]. Moreover, this specific antibody $(\mathrm{Ab})$ response was predictive of malaria morbidity [8]. It was also observed that individuals with $P$. falciparum or Plasmodium vivax infection showed higher IgG response to Anopheles dirus [9] and Anopheles darlingi saliva [10], than uninfected individuals. In addition, one study showed that specific IgG $\mathrm{Ab}$ response to An. gambiae saliva may also be useful for assessing exposure in travellers, and thus could be a biomarker of vector exposure in individuals weakly and transiently exposed to Anopheles bites [11]. Altogether, the specific IgG Ab response to Anopheles salivary proteins represents an immunological marker of human exposure to vector bites and thus a potential indicator of the risks of Plasmodium transmission, as observed for various vector-borne diseases $[12,13]$. With the aim to develop a specific, simple and reproducible biomarker, one synthetic peptide (gSG6-P1) of the An. gambiae (gSG6) protein has been identified and validated as a biomarker candidate of exposure to Afro-tropical malaria vectors [14,15]. Indeed, gSG6-P1 is antigenic, highly specific to Anopheles and it was demonstrated that specific IgG response was especially a relevant biomarker in a context of low and seasonal exposure to malaria vectors [16-18].

This present study aimed i) to appraise the prevalence of $P$. falciparum infection during the dry season in the Senegal River valley and ii) to evaluate the association between IgG responses to gSG6-P1 salivary peptide and different clinical statuses (uninfected, asymptomatic, symptomatic) of $P$. falciparum infection in children. The final objective was to assess the potential use of the gSG6-P1 as a biomarker of P. falciparum infection risk during dry season.

\section{Methods}

\section{Study site and population}

The study was conducted in Northern Senegal, in the Senegal River Valley. This site is a typical sahelian area with less than $400 \mathrm{~mm}$ of rainfall per year. Malaria transmission is described as low, seasonal and occurs mainly during the rainy season between August and October with a rate of infective bites/person/night $<1$ [19].

A longitudinal follow-up was performed from October 2008 to January 2010 in children between one and nine years of age, in Agniam, Niandane, Pendao, Guede and Fanaye, as previously described [18,20]. In the present study, only children present at both the beginning and the end of the dry season of 2009 (January and June) were included in parasitological and immunological analyses. The characteristics of the studied population (number of children, age, and gender) are described in Table 1 . Most of the study population belonged to the Fulani ethnic group.

\section{Ethics statement}

The present study was approved by the National Ethics Committee of the Ministry of Health of Senegal (October 2008; 0084/MSP/DS/CNRS, clinicaltrials.gov/ct2/show/ NCT01545115). Oral and written informed consents 
Table 1 Characteristics of the studied population in the dry season, parasitological and immunological data

\begin{tabular}{|c|c|c|c|}
\hline & Dry season $^{\mathrm{a}}$ & January 2009 & June 2009 \\
\hline $\mathrm{Nb}$ of children & 681 & 371 & 310 \\
\hline Age, mean $\pm S D$ (years) & $5.52 \pm 2.5$ & $5.27 \pm 2.6$ & $5.77 \pm 2.4$ \\
\hline Sex ratio (male/female)(\%) & $341 / 681(50 \%)$ & 188/183 (50.6\%) & $153 / 157(49.4 \%)$ \\
\hline Prevalence of $P$. falciparum infection $(\%)^{b}$ & $98 / 681(14.4 \%)$ & $87 / 371(23.5 \%)$ & $11 / 310(3.5 \%)$ \\
\hline Gametocyte carriage (\%) & $7 / 681(1.0 \%)$ & $3 / 371(1.0 \%)$ & $3 / 310(1.0 \%)$ \\
\hline$\%$ responders $\lg G$ anti-gSG6-P1 ${ }^{c}$ & $102 / 681(14.9 \%)$ & $59 / 371(15.9 \%)$ & $43 / 310(13.9 \%)$ \\
\hline
\end{tabular}

${ }^{a}$ Cumulative data for January and June 2009.

${ }^{\mathrm{b}}$ All $P$. falciparum infection (malaria positive blood smear) with and without symptoms.

${ }^{c}$ Number and percentage (\%) of immune responders of IgG Ab to An. gambiae gSG6-P1 peptide.

were obtained from the parents or the legal guardians of the children.

\section{Study design}

The design of the entomological, parasitological and immunological monitoring (from October 2008 to January 2010, including wet and dry seasons) of each village (Agniam, Niandane, Pendao, Guede and Fanaye) have been previously reported [18]. This present study focused on visits conducted during the dry season (January and June 2009). Exposure to Anopheles bites was extremely low in this area during the dry season and remained similar between villages [18]. Therefore, entomological (Human Biting Rate, HBR), immunological (percentage of IgG responders to gSG6-P1) and parasitological data (parasite prevalence and gametocyte carriage) in the present study represented the mean values of these five villages during the dry season.

\section{Parasitological, immunological and clinical survey}

681 thick blood smears were collected during the two mentioned visits (January and June 2009) to estimate $P$. falciparum prevalence and gametocyte carriage. The slides were transferred to a nearby laboratory, stained with Giemsa and analysed under a microscope by experienced technicians. In parallel, sera collected by finger prick into BD microtainer ${ }^{\circ}$ tubes (Franklin Lakes, New Jersey, USA) were centrifuged and $300 \mu \mathrm{L}$ of serum was collected and stored at $-20^{\circ} \mathrm{C}$ until use for immunological tests by ELISA. Axillary temperature of each participant was also measured.

\section{Study definitions}

According to the $P$. falciparum malaria diagnosis and the clinical status of the disease, children were stratified in three different groups: uninfected (negative malaria blood smear), infected-asymptomatic and infected-symptomatic (positive malaria blood smear, PMBS). Children presenting a PMBS (P. falciparum infection) without any clinical sign, fever (axillary temperature $\geq 37.5^{\circ} \mathrm{C}$ ) and/or a history of fever reported during the corresponding month, were considered infected-asymptomatic; while in the presence of fever and/or history of fever, they were classified as infected-symptomatic.

\section{Entomological data}

Entomological data collected by human landing catches and Pyrethrum spray catches during the follow-up were previously reported and An. gambiae s.l. (An. arabiensis, An. gambiae $S$ and An. gambiae $M$ ) have been described as the predominant species and the only vectors of $P$. falciparum [19]. Other species such as An. funestus, Anopheles wellcomei, Anopheles pharoensis and Anopheles ziemanni were also collected in low densities. At each visit, entomological sampling was performed using four adult volunteers per village (two indoors and two outdoors). Human Biting Rate (HBR) was estimated by the number of bites per person per night (BHN). It was calculated by dividing the number of An. gambiae caught by the total person-night for the period [19].

\section{Salivary peptide gSG6-P1}

The gSG6-P1 peptide was designed using bioinformatics to maximize its Anopheles specificity and its immunogenicity, as previously described $[14,15]$. It was synthesized and purified ( $>95 \%$ ) by Genepep SA (Saint Jean de Védas, France). Peptide was shipped in lyophilized form and then suspended in $0.22 \mu \mathrm{m}$ ultra-filtered water and frozen at $-20^{\circ} \mathrm{C}$ until use.

\section{Evaluation of anti-human IgG level to gSG6-P1 antigen by ELISA}

ELISAs were carried out on sera to quantify IgG response to the gSG6-P1 peptide as previously described [18]. Briefly, Maxisorp plates (Nunc, Roskilde, Danemark) were coated with gSG6-P1 $(20 \mu \mathrm{g} / \mathrm{ml})$ in PBS (Phosphate Buffered Saline). After washing (distilled water + Tween $0.1 \%$ ), each serum were incubated in duplicate at $4^{\circ} \mathrm{C}$ overnight at a $1 / 20$ dilution (in PBS with $1 \%$ Tween). Mouse biotinylated Ab to human IgG (BD Pharmingen, San Diego CA, USA) was incubated at a 1/2000 dilution in PBS with $1 \%$ Tween $\left(1 \mathrm{~h} 30\right.$ at $\left.37^{\circ} \mathrm{C}\right)$ and peroxidase- 
conjugated streptavidin (Amersham, les Ulis, France) was then added $\left(1 / 2,000 ; 1 \mathrm{~h}\right.$ at $\left.37^{\circ} \mathrm{C}\right)$. Colorimetric development was carried out using ABTS (2.2'-azino-bis (3 ethylbenzthiazoline 6-sulfonic acid) diammonium; Sigma, St Louis, MO, USA) in $50 \mathrm{mM}$ citrate buffer (Sigma, $\mathrm{pH}=4$, containing $0.003 \% \mathrm{H}_{2} \mathrm{O}_{2}$ ) and absorbance (OD) was measured at $405 \mathrm{~nm}$.

Individual results were expressed as the $\triangle \mathrm{OD}$ value: $\triangle \mathrm{OD}=\mathrm{ODxODn}$, where ODx represents the mean of individual optical density (OD) value in both wells with gSG6-P1 antigen and ODn the individual OD value for each serum without gSG6-P1 antigen. Specific antigSG6-P1 IgG response were also assayed in non-Anopheles exposed individuals $(\mathrm{n}=12$ - neg; North of France) in order to quantify the non-specific background Ab level and to calculate the specific immune response threshold: $\mathrm{TR}=$ mean $(\Delta \mathrm{ODneg})+3 \mathrm{SD}=0.180$. An exposed individual was then classified as an immune responder (IR) if its $\Delta \mathrm{OD}>0.180$.

\section{Statistical analysis}

Data were analysed with Graph Pad Prism ${ }^{\circ}$ (Graph Pad Software, San Diego, USA). Chi ${ }^{2}$ test was used to compare $P$. falciparum prevalence between January and June, and also between IgG immune responders to gSG6-P1 and non-responders. Mann-Whitney $U$ test was used for the comparison of IgG levels between uninfected and infected-asymptomatic or infected-symptomatic children. The same test was used to compare differences between uninfected and infected children. Differences of IgG response levels between more than three groups were calculated using the non parametric Kruskal-Wallis test. The association between P. falciparum infection and immune responders to gSG6-P1 peptide was calculated using the relative risk (RR), and 95\% confidence intervals (CI) were calculated by the Miettinen method. All differences were considered significant at $\mathrm{p}<0.05$.

\section{Results}

Malaria infection during the dry season

As indicated in Table 1, 681 children have been included during the dry season (371 in January 2009 and 310 in June 2009) among them 304 were present at both visits. Mean age and sex ratio were similar at both visits. Microscopic examination of thick blood smears revealed some $P$. falciparum infection in children during dry season. The prevalence of $P$. falciparum infection was higher in January 2009 (23.5\%) than in June 2009 (3.5\%) ( $\mathrm{p}<0.001, \mathrm{Chi}^{2}$ Test). No difference in P. falciparum infection was observed between children under 5 years of age and the older ones (Additional file 1). Only nine clinical cases of malaria were recorded during both visits, six in January 2009 (1.6\%) and three in June 2009 (1\%). Gametocyte carriage during this period remained very low (1\%). HBR was also very low and was estimated at 0.3 during the cold dry season (January 2009) and 1.52 during the hot dry season (June 2009) [18]. This low exposure to Anopheles bites was confirmed by immunological data that showed $14.9 \%$ of IgG responders to gSG6-P1 (Table 1). In addition, no infected Anopheles were collected during this dry season in the area (Entomological Inoculation Rate, EIR =0) [19].

\section{IgG response levels to gSG6-P1 according to malaria infection}

First, differences of specific IgG response levels to gSG6-P1 salivary peptide were compared between individuals with $P$. falciparum infection (children who presented a PMBS, infected-symptomatic or infected-asymptomatic) and uninfected. Comparison showed that the median of specific IgG Ab level was significantly higher in P. falciparum-infected children compared to uninfected ones during the dry season ( $\mathrm{p}<0.01$, Figure 1A, Mann-Whitney U Test). A similar pattern of specific IgG level according to malaria infection was observed in January $(\mathrm{p}=0.02$, Figure $1 \mathrm{~B})$ and in June $(\mathrm{p}<0.01$, Figure 1C).

\section{lgG response levels to gSG6-P1 according to malaria status}

The specific IgG Ab response to gSG6-P1 was then analysed according to the three different statuses of $P$. falciparum infection (uninfected, infected-asymptomatic and infected-symptomatic) during the dry season (Figure 2; cumulative data for January and June 2009). The antigSG6-P1 IgG levels were significantly different according to the status of $P$. falciparum infection $(\mathrm{p}=0.003$, Kruskal-Wallis Test). Indeed, specific IgG responses were significantly higher in both infected-asymptomatic and infected-symptomatic children compared to uninfected ones $(\mathrm{p}<0.01$ and $\mathrm{p}=0.04$, Mann-Whitney $\mathrm{U}$ test, respectively). But, no statistical difference was observed in IgG $\mathrm{Ab}$ responses between infected-asymptomatic and infected-symptomatic children, $(\mathrm{p}=0.43$, MannWhitney U test). Similar results were obtained when data were analysed separately in January and in June 2009 (Additional file 2). IgG response levels remained higher in infected-asymptomatic children compared to uninfected ones $(\mathrm{p}<0.05)$. Nevertheless, there was no difference either between uninfected and infectedsymptomatic or between infected-asymptomatic and infected-symptomatic.

\section{lgG response levels to gSG6-P1 and P. falciparum infection risk}

To examine if the evaluation of specific IgG Ab levels to gSG6-P1 could be a suitable method to estimate the probability of $P$. falciparum infection during the dry season, i.e. under conditions of low transmission, prevalence of 


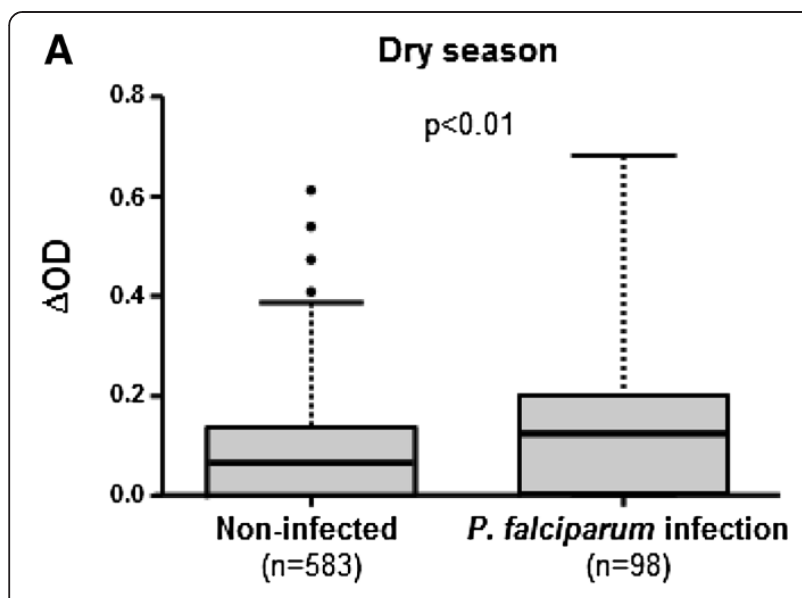

B January 09

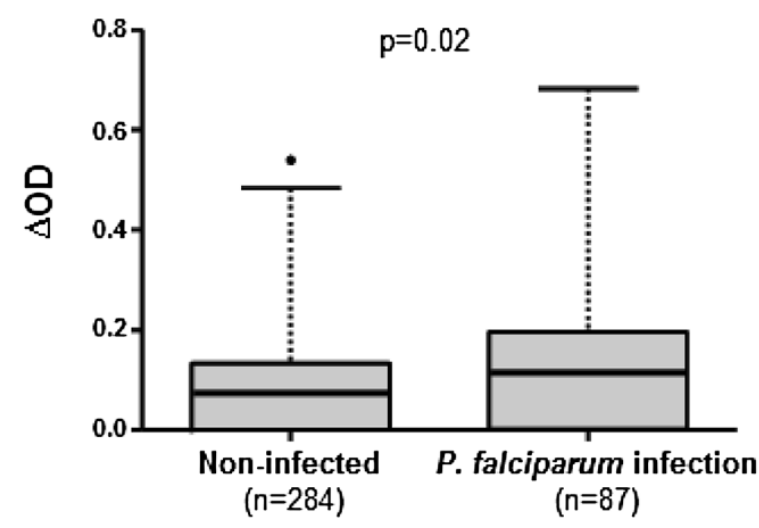

C June 09

응

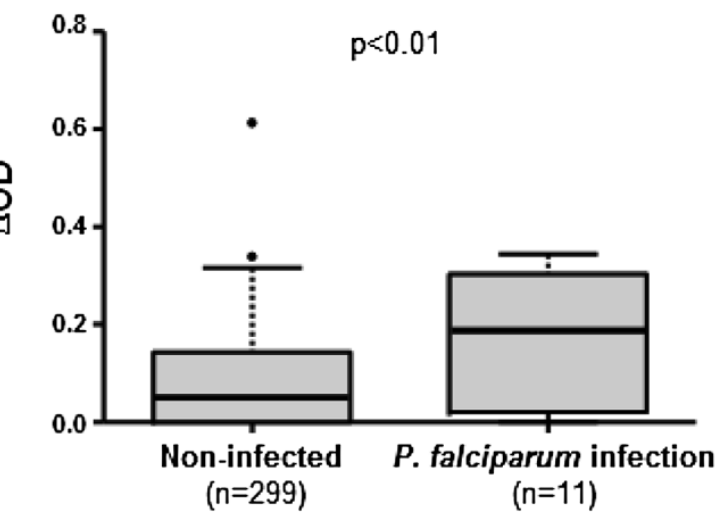

Figure $1 \mathrm{lgG}$ response levels to gSG6-P1 peptide from non-infected and infected children. Box plots indicate lgG response $(\triangle O D)$ values to gSG6-P1 peptide from uninfected individuals and children with $P$. falciparum infection during the whole dry season (cumulative data) (A), in January 09 (B) and in June 09 (C). Boxes display the median $\triangle O D$ value, $25^{\text {th }}$ and $75^{\text {th }}$ percentiles. The whiskers show the $5^{\text {th }} / 95^{\text {th }}$ percentiles and the dots indicate the outliers. The $\mathrm{P}$ value was determined according to the Mann-Whitney U test.

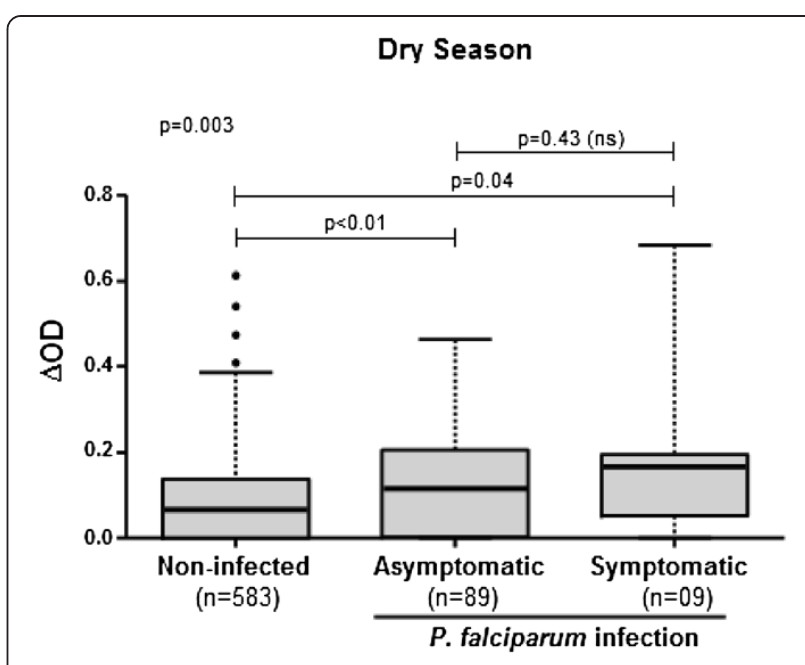

Figure 2 lgG response levels to gSG6-P1 peptide according to malaria status. Box plots show anti-gSG6-P1 lgG level $(\triangle \mathrm{OD})$ between uninfected, infected-asymptomatic and infectedsymptomatic individuals (cumulative data from January and June 2009). Boxes display the median $\triangle O D$ value, $25^{\text {th }}$ and $75^{\text {th }}$ percentiles. The whiskers show the $5^{\text {th }} / 95^{\text {th }}$ percentiles and the dots indicate the outliers. Differences between two or three groups were tested using Mann Whitney test and Kruskal Wallis test, respectively.

P. falciparum infection was compared between immune IgG responders to gSG6-P1 $(\Delta \mathrm{OD}>0.180)$ and non-immune responders $(\Delta \mathrm{OD} \leq 0.180)$ (Figure 3). Positive and significant association between malaria infection prevalence and immune responder to gSG6-P1 was observed. Indeed, the risk of $P$. falciparum infection was $2-7$ times higher in immune responders than in non-responders during the dry season (Figure 3A; relative risk $[R R]=2.504$, 95\% CI: 1.70-3.67, $\mathrm{p}<0.001, \mathrm{Chi}^{2}$ test), in January (Figure 3B; RR $=2.014,95 \% \mathrm{CI}: 1.34-3.00, \mathrm{p}<0.001$ ) and in June (Figure 3C; RR $=7.451,95 \% \mathrm{CI}$ : 3.08-17.97, $\mathrm{p}<0.0001)$. Taken together, these data suggest that positive IgG response to gSG6-P1 could be a pertinent and reliable indicator for suspected $P$. falciparum infection in individuals living in a low transmission context.

\section{Discussion}

In the first part of the study, P. falciparum infection in the human population during the dry season was investigated in the Senegal River Valley reported as a low malaria transmission setting [19]. P. falciparum infection was observed during the dry season and was higher in January $(23.5 \%)$ than in June $(3.5 \%)$. This finding confirmed previous studies in a neighbouring area $[21,22]$ in which a moderate $P$. falciparum infection rate decreases throughout the dry season (December to June) [18].

The paradox observed in this area, between low or no transmission $(E I R=0)$ and high or moderate malaria parasite prevalence, has previously been described in a neighbouring area [21] and elsewhere in Africa [23-25]. 
Figure 3 Plasmodium falciparum prevalence between IgG responders and non-responders to gSG6-P1 peptide. Data present the rate of $P$. falciparum infection according to the two defined immune responder groups: non-responders to gSG6-P1 $(\triangle \mathrm{OD} \leq 0.180)$ and responders to gSG6-P1 $(\triangle \mathrm{OD}>0.180)$ during the whole dry season (cumulative data) (A), in January 09 (B) and in June 09 (C). Statistical significant difference between these two groups is indicated using a Chi ${ }^{2}$ test.

Such an observation raises again the question about the relationships between the EIR indicator and its pertinence, and the prevalence of malaria infection. The absence of infection in Anopheles could be explained by a lack of sensitivity of entomological sampling methods used, in a special context where a very small number of An. gambiae s.l. is collected. Indeed, An. gambiae s.s and $A n$. arabiensis were already proven to be highly anthropophilic and predominant malaria vectors in the Senegal River Valley [19]. Anopheles infection rate of $1.37 \%$ was observed one year later during the dry season [19]. This suggests that malaria transmission could occur during the dry season between November and June corresponding to off-season agriculture. However, its intensity could differ from one year to another [1]. Thus, irrigated fields' crops, annual flood of the river, temporary ponds or ditches could provide breeding sites for remaining Anopheles [19,26,27], and thereby contribute to malaria transmission during the dry season. However, it should be noticed that very few clinical cases of malaria were recorded in this area during the dry season. Indeed, the Senegalese NMCP reported in 2009 less than five malaria cases per 1,000 inhabitants of the River Valley [2]. In our study, most of infected children were asymptomatic carriers of the parasite and the identification of such individuals could be very relevant for a better malaria control, especially during the dry season and in areas of malaria pre-elimination.

In this regard, the present study shows that IgG response to gSG6-P1 was higher in infected-asymptomatic children than in uninfected ones. Statistical significance between infected-asymptomatic and uninfected children was stronger $(\mathrm{p}<0.01)$ when data were cumulated (January and June 2009); probably due to the huge amount of overlap between groups during these two visits. However, IgG response to gSG6-P1 peptide remained significantly higher in infected-asymptomatic children than in uninfected ones when data were analysed separately in January $(\mathrm{p}=0.04)$ and in June $(\mathrm{p}=0.02)$. So, IgG response to $A n$. gambiae gSG6-P1 salivary peptide could be a pertinent biomarker of $P$. falciparum asymptomatic infection. Such a distinction of asymptomatic $P$. falciparum infected individuals compared to uninfected children appears considerable for malaria control in low endemicity areas, and especially during the dry season. Indeed, most of 
infected children are asymptomatic carriers of the parasite and long-term asymptomatic carriage of $P$. falciparum throughout the dry season is commonly observed $[3,4,28]$. Infected-asymptomatic individuals could serve as a crucial reservoir for the parasite and could transmit $P$. falciparum to uninfected Anopheles vectors which remain after the rainy season, contributing to the maintenance of malaria transmission in the dry season and its re-starting after the first rains [5,29]. Therefore, monitoring the IgG response to gSG6-P1 salivary peptide as a biomarker of $P$. falciparum asymptomatic infection could be used as a valuable tool in a malaria pre-elimination context.

In addition, the present study showed that high IgG levels to gSG6-P1 could be predictive indicators of $P$. falciparum infection. This result is in accordance with previous results in Senegal showing that children with subsequently clinical $P$. falciparum malaria presented higher anti-saliva IgG response than did non-infected children [8]. In addition, Andrade et al. [10] have showed that higher anti-An. darlingi saliva Ab response could indicate human exposure to $P$. vivax and biomarker of $P$. vivax in Brazil. Recently, a study in Burkina-Faso showed a positive correlation between malaria incidence and IgG response against the recombinant protein of $A n$. gambiae (gSG6) [30]. In Kenya, it has also been indicated that parasite prevalence was associated with the increase of anti-gSG6-P1 IgG prevalence [31]. Altogether, these results suggest that antisaliva (or protein/peptide) Ab response, previously demonstrated to be biomarker of exposure to Anopheles bites in numerous contexts, could also be a useful indicator of malaria infection and/or morbidity risks in this particular context. It is quite conceivable that salivary peptides could complete in the near future, routine thick blood smears performed in the framework of active case detection of malaria infection or in the evaluation of vector control strategies. Indeed, it was shown that it was possible to assess by ELISA, IgG response to gSG6-P1 on eluates of blood drops collected on blotting paper [16,32,33]. This sampling technique could be performed concomitantly to thick blood smears and would allow making several molecular analyses (DNA sequencing of organisms, modern diagnostic techniques based on biomarkers detection.) on the same sample. This method is therefore suitable for multidisciplinary large-scale studies [34]. Furthermore, it is also conceivable to develop such immunological biomarker by the use of a simple self-test strip, like rapid diagnostic test (RDT). This would rapidly identify people at risk or even asymptomatic malaria infected individuals and begin early treatment. Nevertheless, the use of biomarkers of human exposure to Anopheles bites as an immunological indicator of disease risk remains to be largely studied. For example, there is a lack of a conventional threshold of immune response to salivary proteins/peptide at which an individual is at risk of malaria infection. The intensity of exposure or transmission varies according to the area and season. Despite these limitations, data of the current study indicate the potentiality to use biomarkers of human exposure to vectors as a reliable and useful tool to detect malaria human reservoirs and help predicting malaria risk, and this in a context of very low exposure to vectors, such as malaria preelimination settings. This point appeared to be clearly relevant in regard to the observed results in June (end of dry season with very low exposure to Anopheles bites) where the risk of $P$. falciparum infection was seven times higher in immune responders than in non-responders.

A limitation of this study could be linked to an underevaluation of $P$. falciparum infection prevalence during the dry season, since it was only determined by microscopy examination. However, this gold standard technique for malaria screening has limited sensitivity for detection of very low parasite densities. Indeed, it has been shown that, in areas with low or very low intensity of malaria transmission, sub-microscopic infections were commonly seen $[35,36]$. It would be important to improve this detection using more sensitive methods such as gene amplification by Polymerase Chain Reaction (PCR) [37-39]. Nevertheless, the presence of P. falciparum infection has been revealed with microscopic detection, suggesting that malaria transmission occurs during the dry season in this area.

\section{Conclusions}

During the low-transmission season (dry season), $P$. falciparum infections were observed in the Northern Senegal River Valley. Parasitological data have been correlated with anti-gSG6-P1 IgG responses, indicating that this Anopheles salivary-antigen specific response could be a pertinent epidemiological biomarker of $P$. falciparum exposure during the dry season. Furthermore, the analysis revealed that high levels of gSG6-P1 specific IgG response could be used as an indicator of infection risk and even to discriminate uninfected and infected-asymptomatic carriers of the parasite during the dry season. This biomarker could be very useful for national malaria control programs to identify asymptomatic carriers of $P$. falciparum in low and seasonal transmission areas and, by consequences, in a context of malaria pre-elimination.

\section{Additional files}

Additional file 1: Prevalence of $P$. falciparum infection during the dry season, stratified by age group. The table summarizes $P$. falciparum infection between children under 5 years of age and the older ones during the dry season. 
Additional file 2: IgG response levels to gSG6-P1 peptide according to malaria status. Box plots show gSG6-P1 specific lgG response levels $(\triangle \mathrm{OD})$ according to three $P$. falciparum infection statuses. Boxes display the median $\triangle O D$ value, $25^{\text {th }}$ and $75^{\text {th }}$ percentiles. The whiskers show the $5^{\text {th }} / 95^{\text {th }}$ percentiles and the dots indicate the outliers. Differences between two or three groups were tested using Mann Whitney test and Kruskal Wallis test, respectively.

\section{Competing interests}

The authors declare that they have no competing interests.

\section{Authors' contributions}

ABS participated in field surveys, carried out the immunological assessments, analysed data and drafted the manuscript. JBS, LG, SS, MP, AMS, EH, NF participated in study coordination, field surveys, data collection and microscopic examination. SBC participated to data analysis. GR and FR participated in study design, preparation and writing of the manuscript. All authors contribute to revision the manuscript and read and approved the final version.

\section{Acknowledgements}

We gratefully acknowledge the populations of Agniam, Niandane, Guede, Ndiayene Pendao and Fanaye, and all the health care agents of the district of Podor, for their participation in the study. We also thank Soihibou Guindo (CRB-EPLS) for his technical and useful assistance in the field. ABS was supported by a fellowships provided by CRB-EPLS. This research was supported by the Biomedical Research Center Espoir Pour La Santé (EPLS) and the Research Institute for Development (IRD-Direction de la Valorisation au Sud) with a financial participation of the FSD "Fond Social de Développement", (BIOMARQPAL ref 2008-5901) Embassy of France in Senegal.

\section{Author details}

${ }^{1}$ Centre de Recherche Biomédicale Espoir Pour La Santé, 269 Route de la corniche, Sor, BP: 226, Saint-Louis, Sénégal. ²Département de Biologie Animale, Laboratoire de parasitologie générale, Université Cheikh Anta Diop, Dakar, Sénégal. ${ }^{3}$ CIIL, Inserm U1019, CNRS UMR 8204, Université Lille Nord de France, Institut Pasteur de Lille, 1 rue du Pr. Calmette, 59019 Lille cedex, France. ${ }^{4}$ Institut de Recherche pour le Développement, UMR 224 MIVEGEC, 911 avenue Agropolis, BP: 64501F-34394 Montpellier, France. ${ }^{5}$ Centre de Recherche Entomologique de Cotonou, BP: 4414, Cotonou RP 01, Bénin.

Received: 26 June 2013 Accepted: 27 August 2013

Published: 30 August 2013

\section{References}

1. Ministry of Health and Prevention of Senegal: National Strategic Plan 2011-2015. Dakar: National Malaria Control Programme; 2010.

2. Ministry of Health and Prevention of Senegal: Statistical report 2009. Dakar: National Malaria Control Programme; 2010.

3. Babiker HA, Abdel-Muhsin AM, Ranford-Cartwright LC, Satti G, Walliker D: Characteristics of Plasmodium falciparum parasites that survive the lengthy dry season in eastern Sudan where malaria transmission is markedly seasonal. Am J Trop Med Hyg 1998, 59:582-590.

4. Hamad AA, Nugud Ael H, Arnot DE, Giha HA, Abdel-Muhsin AM, Satti GM, Theander TG, Creasey AM, Babiker HA, Elnaiem DE: A marked seasonality of malaria transmission in two rural sites in eastern Sudan. Acta Trop 2002, 83:71-82.

5. Zwetyenga J, Rogier C, Spiegel A, Fontenille D, Trape JF, Mercereau-Puijalon $\mathrm{O}$ : A cohort study of Plasmodium falciparum diversity during the dry season in Ndiop, a Senegalese village with seasonal, mesoendemic malaria. Trans R Soc Trop Med Hyg 1999, 93:375-380.

6. Paul RE, Diallo M, Brey PT: Mosquitoes and transmission of malaria parasites - not just vectors. Malar J 2004, 3:39

7. Richel R, Sondaz D, Philip J, Calvet F, Daoud W: Le bassin versant du fleuve Sénégal, situation sanitaire en 2010. Med Trop 2010, 71:223-228.

8. Remoue F, Cisse B, Ba F, Sokhna C, Herve JP, Boulanger D, Simondon F: Evaluation of the antibody response to Anopheles salivary antigens as a potential marker of risk of malaria. Trans R Soc Trop Med Hyg 2006, 100:363-370.
9. Waitayakul A, Somsri S, Sattabongkot J, Looareesuwan S, Cui L, Udomsangpetch R: Natural human humoral response to salivary gland proteins of Anopheles mosquitoes in Thailand. Acta Trop 2006, 98:66-73.

10. Andrade BB, Rocha BC, Reis-Filho A, Camargo LM, Tadei WP, Moreira LA, Barral A, Barral-Netto M: Anti-Anopheles darlingi saliva antibodies as marker of Plasmodium vivax infection and clinical immunity in the Brazilian Amazon. Malar J 2009, 8:121

11. Orlandi-Pradines E, Almeras L, de Denis Senneville L, Barbe $S$, Remoue F, Villard C, Cornelie S, Penhoat K, Pascual A, Bourgouin C, Fontenille D, Bonnet J, Corre-Catelin N, Reiter P, Pages F, Laffite D, Boulanger D, Simondon F, Pradines B, Fusai T, Rogier C: Antibody response against saliva antigens of Anopheles gambiae and Aedes aegypti in travellers in tropical Africa. Microbes Infect 2007, 9:1454-1462.

12. Billingsley PF, Baird J, Mitchell JA, Drakeley C: Immune interactions between mosquitoes and their hosts. Parasite Immunol 2006, 28:143-153.

13. Remoue F, Cornelie S, Ngom A, Boulanger D, Simondon F: Immune responses to arthropod bites during vector-borne diseases, Update in tropical immunology. Herala, India: Fort P.O. Trivandrum; 2005:377-400.

14. Poinsignon A, Cornelie S, Mestres-Simon M, Lanfrancotti A, Rossignol M, Boulanger D, Cisse B, Sokhna C, Arca B, Simondon F, Remoue F: Novel peptide marker corresponding to salivary protein gSG6 potentially identifies exposure to Anopheles bites. PLOS One 2008, 3:e2472.

15. Poinsignon A, Samb B, Doucoure S, Drame PM, Sarr JB, Sow C, Cornelie S, Maiga S, Thiam C, Rogerie F, Guindo S, Hermann E, Simondon F, Dia I, Riveau G, Konate L, Remoue F: First attempt to validate the gSG6-P1 salivary peptide as an immuno-epidemiological tool for evaluating human exposure to Anopheles funestus bites. Trop Med Int Health 2010, 15:1198-1203.

16. Drame PM, Machault V, Diallo A, Cornelie S, Poinsignon A, Lalou R, Sembene M, Dos Santos S, Rogier C, Pages F, Le Hesran JY, Remoue F: IgG responses to the gSG6-P1 salivary peptide for evaluating human exposure to Anopheles bites in urban areas of Dakar region. Senegal. Malar J 2012, 11:72.

17. Poinsignon A, Cornelie S, Ba F, Boulanger D, Sow C, Rossignol M, Sokhna C, Cisse $B$, Simondon F, Remoue F: Human IgG response to a salivary peptide, gSG6-P1, as a new immuno-epidemiological tool for evaluating low-level exposure to Anopheles bites. Malar J 2009, 8:198.

18. Sagna AB, Sarr JB, Gaayeb L, Drame PM, Ndiath MO, Senghor S, Sow CS, Poinsignon A, Seck M, Hermann E, Schacht AM, Faye N, Sokhna C, Remoue F, Riveau G: gSG6-P1 salivary biomarker discriminates micro-geographical heterogeneity of human exposure to Anopheles bites in low and seasonal malaria areas. Parasit Vectors 2013, 6:68.

19. Ndiath MO, Sarr JB, Gaayeb L, Mazenot C, Sougoufara S, Konate L, Remoue F, Hermann E, Trape JF, Riveau G, Sokhna C: Low and seasonal malaria transmission in the middle Senegal River basin: identification and characteristics of Anopheles vectors. Parasit Vectors 2012, 5:21.

20. Gaayeb L, Sarr JB, Ndiath MO, Hanon JB, Debrie AS, Seck M, Schacht AM, Remoue F, Hermann E, Riveau G: Seroprevalence of pertussis in Senegal: a prospective study. PLoS One 2012, 7:e48684.

21. Dia I, Konate L, Samb B, Sarr JB, Diop A, Rogerie F, Faye M, Riveau G, Remoue F, Diallo $M$, Fontenille D: Bionomics of malaria vectors and relationship with malaria transmission and epidemiology in three physiographic zones in the Senegal River Basin. Acta Trop 2008, 105:145-153.

22. Sarr JB, Remoue F, Samb B, Dia I, Guindo S, Sow C, Maiga S, Tine S, Thiam C, Schacht AM, Simondon F, Konate L, Riveau G: Evaluation of antibody response to Plasmodium falciparum in children according to exposure of Anopheles gambiae s.l. or Anopheles funestus vectors. Malar J 2007, 6:117.

23. Beier JC, Killeen GF, Githure Jl: Short report: entomologic inoculation rates and Plasmodium falciparum malaria prevalence in Africa. Am J Trop Med Hyg 1999, 61:109-113.

24. Hay SI, Guerra CA, Tatem AJ, Atkinson PM, Snow RW: Urbanization, malaria transmission and disease burden in Africa. Nat Rev Microbiol 2005, 3:81-90.

25. Robert V, Le Goff G, Andrianaivolambo L, Randimby FM, Domarle O, Randrianarivelojosia M, Raharimanga V, Raveloson A, Ravaonjanahary C, Ariey F: Moderate transmission but high prevalence of malaria in Madagascar. Int J Parasitol 2006, 36:1273-1281.

26. Guthmann JP, Llanos-Cuentas A, Palacios A, Hall AJ: Environmental factors as determinants of malaria risk. A descriptive study on the northern coast of Peru. Trop Med Int Health 2002, 7:518-525.

27. Koita OA, Sangare L, Sango HA, Dao S, Keita N, Maiga M, Mounkoro M, Fane Z, Maiga AS, Traore K, Diallo A, Krogstad DJ: Effect of seasonality and 
ecological factors on the prevalence of the four malaria parasite species in northern mali. J Trop Med 2012, 2012:367160.

28. Males S, Gaye O, Garcia A: Long-term asymptomatic carriage of Plasmodium falciparum protects from malaria attacks: a prospective study among Senegalese children. Clin Infect Dis 2008, 46:516-522.

29. Alves FP, Gil LH, Marrelli MT, Ribolla PE, Camargo EP, Da Silva LH: Asymptomatic carriers of Plasmodium spp. as infection source for malaria vector mosquitoes in the Brazilian Amazon. J Med Entomol 2005, 42:777-779.

30. Stone W, Bousema T, Jones S, Gesase S, Hashim R, Gosling R, Carneiro I, Chandramohan D, Theander T, Ronca R, Modiano D, Arca B, Drakeley C: IgG responses to Anopheles gambiae salivary antigen gSG6 detect variation in exposure to malaria vectors and disease risk. PLoS One 2012, 7:e40170.

31. Badu K, Siangla J, Larbi J, Lawson BW, Afrane Y, Ong'echa J, Remoue F, Zhou G, Githeko AK, Yan G: Variation in exposure to Anopheles gambiae salivary gland peptide (gSG6-P1) across different malaria transmission settings in the western Kenya highlands. Malar J 2012, 11:318.

32. Drame PM, Diallo A, Poinsignon A, Boussari O, Dos Santos S, Machault V, Lalou R, Cornelie S, Lehesran JY, Remoue F: Evaluation of the effectiveness of malaria vector control measures in urban settings of dakar by a specific anopheles salivary biomarker. PLoS One 2013, 8:e66354.

33. Drame PM, Poinsignon A, Besnard P, Cornelie S, Le Mire J, Toto JC, Foumane V, Dos-Santos MA, Sembene M, Fortes F, Simondon F, Carnevale $P$, Remoue F: Human antibody responses to the Anopheles salivary gSG6-P1 peptide: a novel tool for evaluating the efficacy of ITNs in malaria vector control. PLoS One 2010, 5:e15596.

34. Pomelova VG, Osin NS: Prospects of the integration of dry blood spot technology with human health and environmental population studies. Vestn Ross Akad Med Nauk 2007:10-16. http://pubget.com/paper/18225500.

35. Okell LC, Bousema T, Griffin JT, Ouedraogo AL, Ghani AC, Drakeley CJ: Factors determining the occurrence of submicroscopic malaria infections and their relevance for control. Nat Commun 2012, 3:1237.

36. Okell LC, Ghani AC, Lyons E, Drakeley CJ: Submicroscopic infection in Plasmodium falciparum-endemic populations: a systematic review and meta-analysis. J Infect Dis 2009, 200:1509-1517.

37. Berry A, Benoit-Vical F, Fabre R, Cassaing S, Magnaval JF: PCR-based methods to the diagnosis of imported malaria. Parasite 2008, 15:484-488.

38. de Monbrison F, Angei C, Staal A, Kaiser K, Picot S: Simultaneous identification of the four human Plasmodium species and quantification of Plasmodium DNA load in human blood by real-time polymerase chain reaction. Trans R Soc Trop Med Hyg 2003, 97:387-390

39. Hanscheid T, Grobusch MP: How useful is PCR in the diagnosis of malaria? Trends Parasitol 2002, 18:395-398.

doi:10.1186/1475-2875-12-301

Cite this article as: Sagna et al:: Plasmodium falciparum infection during dry season: IgG responses to Anopheles gambiae salivary gSG6-P1 peptide as sensitive biomarker for malaria risk in Northern Senegal. Malaria Journal 2013 12:301.

\section{Submit your next manuscript to BioMed Central and take full advantage of:}

- Convenient online submission

- Thorough peer review

- No space constraints or color figure charges

- Immediate publication on acceptance

- Inclusion in PubMed, CAS, Scopus and Google Scholar

- Research which is freely available for redistribution

Submit your manuscript at www.biomedcentral.com/submit
C Biomed Central 